\title{
Higher Education Research Methodology-Literature Method
}

\author{
Guijuan Lin \\ Xiamen Software College \\ No. 1199 Sunban South Road, Jimei District, Xiamen, Fujian Province 361012, China \\ Tel: 86-592-5053678Ｅ-mail:et9898@163.com
}

\begin{abstract}
Starting by a brief introduction of literature method, this paper raises the question of how to choose representative research literatures. For this question, Professor Hongcai Wang points out the root of this problem and the strategies to resolve it and provides the ways to choose representative literatures. Through analyzing and summarizing, this paper comes to a conclusion of "how to choose representative research literature".
\end{abstract}

Keywords: Literature method, Representative, Research literatures, Root, Strategies, Ways

\section{Literature methodology}

Literature research methodology is to read through, analyze and sort literatures in order to identify the essential attribute of materials. Its significant difference from other methodologies is that it does not directly deal with the object under study, but to indirectly access to information from a variety of literatures, which is generally referred to as "non-contact method." Literature materials are the crystallization of wisdom, are the ocean of knowledge, have important values for the development of human society, history, culture and research scholars. Education researches shall fully share information, conduct literature researches to grasp sources of relevant researches and scientific developments and to understand what our predecessors have achieved and the progress made by other researchers. However, in the ocean of knowledge of such a vast amount of information, how should we choose representative literatures?

\section{How to choose representative research literatures}

As we write dissertations, we often encounter the problem of what materials are representative literatures.

\subsection{The root of the problem.}

According to Professor Hongcai Wang, the root of this problem, for researchers, is the lack of qualitative analysis of the literatures.

Zhenguo Yuan points out that "literature research methodology" include non-structured qualitative analysis and structured quantitative analysis. They access to and process information contained in literatures from different perspectives. Generally speaking, literatures are descriptions of the nature, functions and characteristics of objects. These qualitative descriptions seldom show the variable relationship of research subject, so researchers tend to apply logical reasoning to explore the logical relations among objects rather than the quantity relationship.

In general, qualitative analysis is to classify information contained in literatures, to select typical examples to re-organize and come to conclusion on the basis of qualitative description. The qualitative analysis of literatures has special values in distinguishing the past trends and forecasting future models.

Qualitative research of literatures does not focus on the quantity and completeness of literature materials. It focuses on personal literature researches and chooses small samples or characteristics of cases according to the interests of the researches and subject requirements.

Qualitative analysis is to study whether the objects under researches have certain nature or to study chance reasons and process analysis. The base of qualitative analysis is philosophy method. It compares the similarities and differences between things through reasoning, sums up the types of things and grasps the rules of things. The common reasoning process of qualitative analysis is as follows: what is the problem? What is the definition of the concept? What are the facts? What is the reason? What is the conclusion of the study? Qualitative analysis commonly applies seven methods, respectively,: causal analysis, comparative analysis, conflicts analysis, the results and functional analysis, induction and deduction, analysis and synthesis and scientific abstract method.

\subsection{Strategies to overcome the problem}

Professor Hongcai Wang provides three strategies to overcome this problem, to talk with scholars, to identify the grade of literatures and to enhance theory knowledge

After we have collected as many literatures as possible related to our research subject, we must sort them out and analyze to determine what representative research literatures are and what general references are. In the determination 
of the representative literatures, several principles must be followed: One is purpose: Literature materials must be pertinent, valuable for the subject under research, are the theoretical base or background information of the research subject and are typical examples. Researchers could use these materials to find out the past trends and to predict future relevant to these materials. The second is. Authority: The author of the literature has reputation or is representative in this research field, or is academic leader, or authority editor of key discipline research bases, and has published articles in national famous magazines. The authority of literature can also be known by identifying how many times the literature has been cited. Under normal circumstances, only literature with authority could earn trust and be given references. The third one is effectiveness. Research materials are valuable for the research subject. The values are reflected through being good for abstracting, being beneficial for obtaining arguments, being conducive to the formation of research thoughts. Therefore, research data must be accurate, comprehensive, profound and typical, not out of date. The stronger the effectiveness is, the more likely the true scientific conclusions could be achieved. The literature then is representative. The fourth one is reliability: The reliability of literature includes authenticity of the literature and the reliability of the contents. When researchers refer to certain literature, they must first make sure whether these literatures are authentic, or fakes or modified, especially for historical documents describing issues. Because the formation time of them is far from our times, it is hard to determine its authenticity. After identifying the authentity of literatures, we must also verify whether the facts or data recorded in the literature are reliable, such as whether the literature faithfully records the truth and whether the concepts and date in the literature and data are accurate, and so on.

Then, how to choose representative research literatures? The following ways could be applied.

\subsubsection{To interview scholars}

To interview scholars and ask them to recommend representative research literatures is a rather direct and efficient way. For example, one of my teachers recommends us to read several books which are all valuable for education researches, which are Encyclopedia for Education Researches, Education Researches Handbook, and Education Researches Comments. These literatures are valuable for us to conduct education researches are representative.

\subsubsection{To identify the grade of literatures}

Under the premise of identifying the grade of literature, we should first explain how to identify the authenticity of literatures. Methods of identifying literature include two ways, respectively, external audit and internal audit. External audit is to identify the authenticity of literatures. We should identify the authenticity of the version by comparing the book's presentation style with contemporary publications; On the other hand, we can also examine whether the contents of the book describe the facts that occur after the book is written since. We should also identify the authenticity of authors. Three ways are generally used. The first one it to analyze other works of the author to know the language styles of the author and then check whether the literature on hand has same language style. The second is to analyze whether the style of the literature is consistent. The third is whether the idea of literature and logical point of view is consistent. Internal audit refers to the identification of the authenticity of contents. Main methods include: first, mutual prove of words. If there are inconsistencies between the events described, we should verify it. Secondly, we could use real object to literature in words. We could check whether the text description is consistent with physical evidence. The third is to compare the contents of the documents with historical background to see if it's consistent with the contemporary political and cultural background. The fourth is to study of the life, point of view, basic thoughts of the author and the specific environment when the literature is formed to determine objectivity and tends of the author. In short, internal audit and external audit both make comparison to identify the authenticity of contents in order to collect quality literature.

To identify the authenticity of the documents, we can grade collected materials. According to the content processing ways, education literature could be divided into zero grade, first grade, second grade and third grade. Zero grade literature is usually called the first-hand literature and is the first description of events or experiences and is written by persons who experience the event and is the most primitive original documents, not published or modified, such as the letter, draft, discussion draft, or original record. First grad literature generally refers to works of the record of the incident, research results, new knowledge and new technology monographs, papers, investigation reports and other literature. Literature is also referred to as a secondary literature, is the evidence description reports or materials processed once by actual witnesses and participants in the events. It includes books, newspapers, periodicals, research reports, and government publications and so on. The second grade literature is also known as the retrieval of documents. It refers to literatures that process and sort books and papers to summarize the main points and to put them in system with some methods, such as the bibliography, titles, abstracts, indexes and other publications. Such documentation will be distributed in a systematic literature, entries. Third grade literature is also known as the reference literature. It systematically sorts the first grade literature and make summary based on the second grade literatures. Such literature is different from the original literature, and also different from the secondary literature. It has subjective nature and reflects the authors opinion on the first grade literature and is the comprehensive research results of many first grade literature, such as the dynamic synthesis of educational research, teaching thematic commentary, teaching progress 
report, which is therefore called as the third grade literature.

\subsubsection{To enhance theory knowledge}

How to choose representative research literature? On this issue, we must not only mater above-mentioned points; we should also study the fundamental theory knowledge of literature methodology. How are we going to collect the literature? To find valuable literatures for certain research subject in the vast literature ocean, we must master effective method to collect the documents. First of all, researchers should determine the scope and nature of the research subject and fix the direction of the search. Secondly, researchers must know education journals and periodicals both at home and abroad, understand domestic and foreign types of educational books, pictures, audio and video, electronic data and statistics overview and know from where could these materials be obtained. Finally, researchers must be familiar with literature index and directory classification method and master the basic skills of literature retrieval.

Then, we must sort and analyze the large number of literatures collected. How to handle it? The sorting of the literature refers to the process in which researchers conduct creative analysis, comparison and summarizing of literatures that are collected and are identified. Through this kind of reasoning, researches form scientific understanding of research subject. The specific methods of literature sorting mainly apply logical analysis, judging, reasoning, synthesis and dialectical thinking, etc., to make fact judgment from the materials or to summarize some rules or principles to sort these materials.

After sorting literatures, we should analyze the literatures. As raised by Zhenguo Yuan, literature research methods include non-structured qualitative analysis methods and structured quantitative analysis. Non-structured qualitative analysis has been illustrated. Structured quantitative analysis is also known as content analysis. Content analysis is to systematically and objective quantitative literature contents and to make description.. For example, when a reader wants to buy a book, he or she will first look at the directory, read an article and take a look at the keywords. These are a kind of intuitive content analysis. Some educators have conducted researches on Education Science by Kaiipob. It is found that Kaiipob mentioned "the transfer of knowledge" more than 100 times and only mentioned "the development of intelligence" only 10 times. Therefore, he put forward that Kaiipob's education science is a education science of imparting knowledge. This study is the use of the content analysis. The characteristics of content analysis are intuitive, objective, systematic, and quantitative. The nature of content analysis is to describe literature in words by data, which is good for formal hypothesis application, for scientifically sampling, and for the application of computers to do literature researches. Content analysis is mainly used in trend analysis, comparative analysis and intention analysis.

\section{Summary}

For the problem of how to choose representative research literatures, we make the following summary.

3.1 To master fundamental theory knowledge, including qualitative analysis, literature grade identifying, literature collection methods, literature sorting and contents analysis.

\subsection{To identify representative literatures based on fundamental knowledge}

3.2.1 The literatures written by experts or scholars from national key discipline research bases

3.2.2 Comment literatures used by discipline annul report or discipline development status meeting

3.2.3 Literatures written by discipline leading scholars

\subsubsection{Literatures often cited}

3.2.5 Literature of time effectiveness reflecting the current development of research fields

3.2.6 Comments by experts on relevant issues of literature and timely learn the authority of literatures.

\section{References}

Li, Fang. Modern Education scientific research methodology. Guangzhou: Guangdong Higher Education Press.

Wang, Shouheng. Basis of education scientific research methodology. Hefei: Anhui University Press.

Wiersman, William. Introduction to educational research methods. Translated by Zhenguo Yuan. Education and Science Press.

Yang, Xiaowei. Fundamentals and methods of education researches. Shanghai: Huadong Normal University Press. Yuan, Zhenguo. Education research methodology. Higher Education Press. 